\title{
THE SPACE DENSITY OF FAINT M-DWARFS
}

\author{
J. H. OORT
}

The Observatory, Leiden, The Netherlands

Donna Weistrop, a student of Maarten Schmidt's, has found evidence for an unexpectedly high space density of very faint M-dwarfs. This was confirmed by Murray and Sanduleak. The space density inferred from these investigations is comparable to, or possibly higher than, the local gas density. Because there is evidence that the stars concerned are young this appears to present a problem.

As the investigation by Murray and Sanduleak is the simpler and more direct of the two I confine my discussion to this. The authors determined proper motions for $21 \mathrm{M}$ dwarfs down to $17^{m}$ found in an objective-prism survey at the Warner and Swasey Observatory in regions close to the North Galactic Pole. The distance of the stars can be inferred from the reflection of the solar motion. Assuming the 'basic' solar motion they found $\langle\pi\rangle=0.021$ and a space density of $0.23 \mathrm{pc}^{-3}$ for these stars, corresponding with a mass density of about $0.04 M_{\odot} \mathrm{pc}^{-3}$. This is exceedingly high, in view of the fact that the average gas density near $z=0$ is only $0.03 M_{\odot} \mathrm{pc}^{-3}$. The problem is aggravated by the fact that the stars have low velocities, the dispersion in velocity in one coordinate coming out $\pm 10 \mathrm{~km} \mathrm{~s}^{-1}$. This is considerably lower than the velocity dispersion of A-type stars, and shows that the stars are young, perhaps about $10^{8} \mathrm{yr}$. How is it then that the gas has not been completely used up long ago?

I believe one cannot discard this conclusion on the ground of inaccuracy of the proper motions; on the average the motions are considerably higher than the probable errors. This is further confirmed by independent data derived by Luyten. Moreover, the entirely different investigation by Donna Weistrop led to very similar, partly even higher densities.

The difficulty can be somewhat relieved by using the 'standard' solar motion instead of the 'basic' solar motion used by Murray and Sanduleak. If the stars are quite young they may be thought to follow the motion of the interstellar medium which gives a higher solar velocity. If we do this, we find a density of $0.016 M_{\odot} \mathrm{pc}^{-3}$, therefore about half the present gas density. The velocity dispersion is raised to $\pm 13 \mathrm{~km} \mathrm{~s}^{-1}$.

But even such a density seems inacceptable for a general average density. A possible way out of this dilemma is that we are seeing a region where the density is considerably higher than the average. This might be the case if we happen to be inside an interstellar cloud.

\section{DISCUSSION}

Murray: I must thank Prof. Oort for this very clear exposition of the work by Sanduleak and myself. I fully agree that the choice of the basic solar motion for estimating the mean parallax was quite arbitrary; if we had chosen a larger motion, the mean parallax, and hence the density, would have 
been smaller. But whatever solar motion one adopts the transverse velocity dispersions appear to be relatively small. Although we only have kinematic data for some twenty stars, Sanduleak actually found 1200 stars in 120 sq deg near the North Galactic Pole; this is not an insignificant sample. It appears, however, that there may be differences between the two galactic polar caps; thus we may be seeing a localized phenomenon. Recent photoelectric photometry in four colours by D. H. P. Jones confirms that probably all the stars in the kinematic sample are in fact dwarfs.

Luyten: In connection with the Murray-Sanduleak stars I might add that whereas their distances were derived from kinematics, I remeasured all their motions and found good agreement except for the seven very small motions. I also redetermined their magnitudes and found them $0.43^{\mathrm{m}}$ fainter than those of Murray and Sanduleak. Then all you need is to shift the spectra 0.06 of a spectral class earlier, i.e. from M3 to M2.4 and there is no (?) more discrepancy with my previous data. I would again emphasize the danger of drawing such far-reaching conclusion from such minuscule samples.

Oort: In my communication I made no use of the magnitudes to estimate the mean distances. This was derived from the reflection of the solar motion. 